\title{
The Design of Autism Content Management Learning System (ACMLS) Framework
}

\author{
Khaled Ismail $^{1}$, Nazean Jomhari ${ }^{2}$ \\ Faculty of Computer Science and Information Technology, University of Malaya, Malaysia \\ 11k.ismail@siswa.um.edu.my, ${ }^{2}$ nazean@um.edu.my
}

\begin{abstract}
The prevalence of autism in children in the world is estimated as one per 62 children, higher levels reported in some countries. These children experience significant problems with the development of social, behavioural and verbal and non-verbal communication skills. The skills impairment levels varies from an individual to another and that made teaching autistics a challenge for caregivers such as teachers and relatives. Hence, there are quite a number of frameworks of a software learning systems which focus on gaining the children's attention using representational visual illustration as a learning method instead of the textual form. However, majority of these tools are lacking the personalisation ability to suite everyone in the spectrum.

Assistive technology offers an alternative way to attract children with autism to use. Therefore, this research is proposing Autism Content Management Learning System (ACMLS) Framework to assist caregivers to produce, design and fine-tune or customise the learning materials appropriately so that the system interface and the materials are suitable for every individual in the spectrum according to each child personal profile aiming to make learning attractive and to contribute in improving their social, communication and behavioural skills and nonetheless, their attention level to the delivered educational topics.
\end{abstract}


The ACMLS framework design adopts four main components which are: (1) Design component: which covers the visual design, design principles and the mental model of the children with autism. (2) Technology component: which covers the assistive technology tools and the architecture of the ACMLS system. (3) Education component: Which covers the learning objectives, styles, strategies, methods and the cognitive model. (4) Participants component: which covers the main participants who're playing a role in the ACMLS framework such as: caregivers and children with autism.

Keywords: children with autism, caregivers, personalised learning, intervention.

\section{I.1 Introduction}

This chapter presents the description of the proposed framework and its main four components such as: technology, education, design and participants whether (Learner, teacher and parents). According to the outcome from preliminary study described in chapter 2, which showed the need for a prospective need to implement a systematic framework contains a combination of visual elements for presenting learning content from the CCI perspective instead of the manual materials. Elements such as colours, images, layouts. ACMLS framework will provide conceptual and practical model as a user guide for a future learning systems development targeting the children with autism in different fields and aspects.

\subsection{ACMLS Framework Components}

The proposed Autism Management Contents Learning System (ACMLS) framework as mentioned earlier consists of four main components which are: Education or learning, Participants, Design and Technology. These components were derived after the preliminary study from the children profiles or biography, learning style, learning approaches for 
autistics, design principles as well as the CCI concepts and theories. Each component in figure 5.1 will be discussed and described in details. 


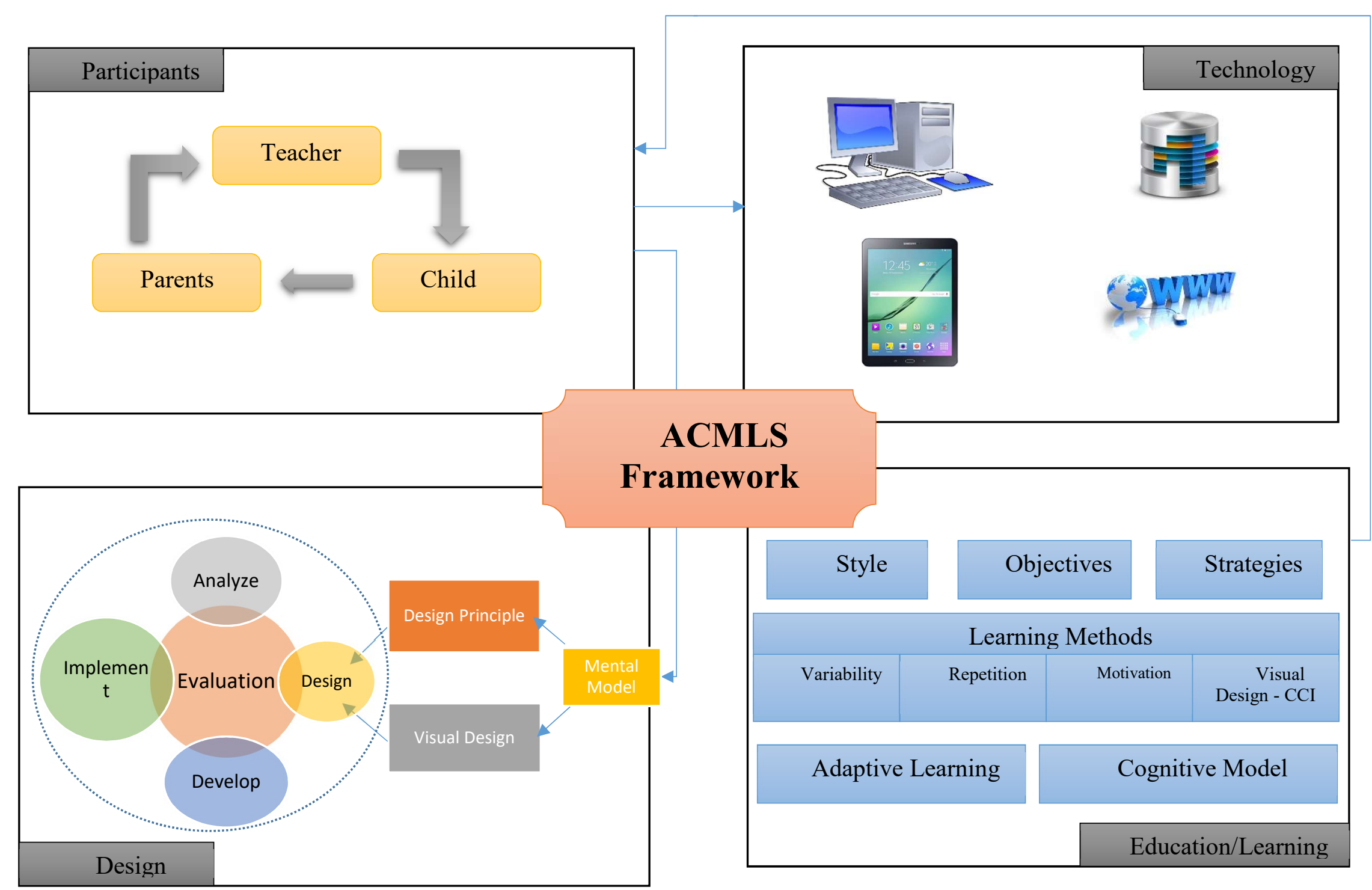

Figure 0.1: ACMLS Framework 


\subsubsection{Participants Component}

$\underline{\text { Participants }}$ The concept of participant in this research has been particularly concerned with the developmental progress of the individuals with autism in their lifelong venture, which is learning for intrinsic reasons. As well as helping the teacher in school and the parents at home to focus on implementing the educational materials based on the curriculum and their daily life routine.

This component consists of the factors that can assist the teachers and parents at home and most importantly influence the children with autism particularly the development fulfilment concern at all stages in their lifelong. More so, the literature review, interviews and observations during the preliminary study helped the researcher to identify the methods and strategies which support gaining the attention during the learning activities.

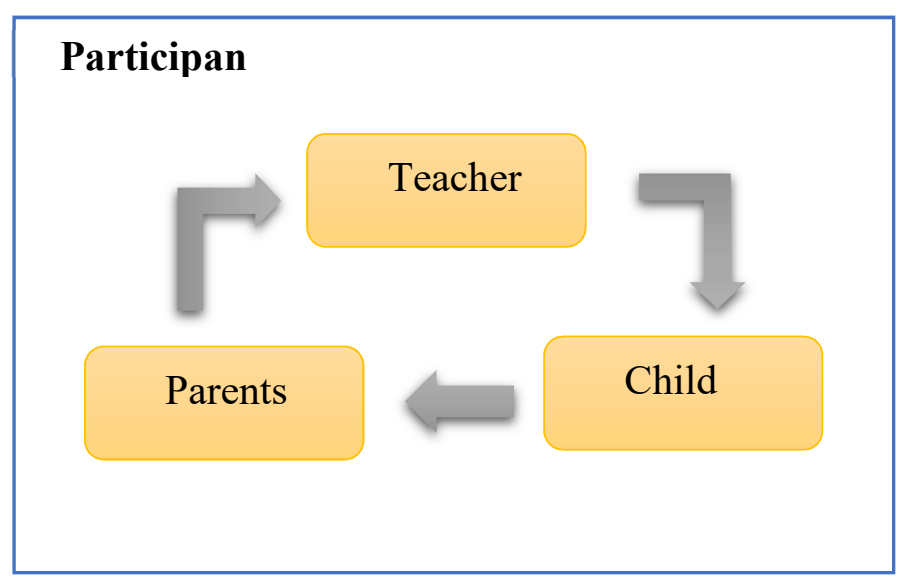

Figure 0.2: Participants component

In addition to that, with a vary individuals with autism skills set and learning requirements, the traditional approaches APA and TEACCH section 3.4.1.2 would be lack of productivity in the learning environment specially when we have a large number of enrolment, and that encouraged 
the researcher to adopt the adaptive learning approach in this research which can be adapted for every individual learning needs targeting a more productive environment for all types of learners.

From the beginning of the study and as mentioned in the literature review chapter as well as earlier in this chapter, we identified the main users of ACMLS framework and they can be divided into two main categories: First category is the learner which is children with autism and second category is the educators such as teachers at schools and parents at home.

From observations during the preliminary study, we found that it is important for those learners (Children with autism) to work closely with their teachers which use learning context reinforcement in most learning activities. Hence, the targeted environment is extended to include educator as another main user of the framework.

\subsubsection{User Profile}

The user profile in ACMLS is a collection of personal data, abilities, education level, and learning style preference. This user profile will be considered as the user identity. This will help in personalising the framework in terms of the colour scheme, font size, sounds and avatars to suite each user based on their profiles. These values can be set upon sign-up to the framework by the teacher or the parent in the user profile page.

After submitting the profile data, and if all information provided are valid then it will be stored in the database and server sends a conformation message presenting the successful sign up process to the framework. These values of the user profile will be reflected on the user interface screen such as font size, colour scheme as well as the avatar used as animation guidance for the learners. 
Lastly, hence the learner's personal privacy matters, therefore, the learner profile data will protected against unauthorized access to the system, and users are required to have login credentials for a secured login.

\subsubsection{Teacher Role}

Is to identify the weaknesses or the areas to improve in every child, and implemented into the system in such away. The system look and feel must be customised to be comfortable to each child. The teacher should input the educational materials or topics into the system from the control panel section. At the end of every assessment the teacher should be able to gather the system feedback and the child progress for evaluation purpose.

\subsubsection{Children with Autism Role}

The children with autism are considered the end user in our system, they're the person under the spot light who is going to use the educations materials which been entered into the system by either the parent or the teacher. The child can access the system from anywhere at any time.

By adapting the adaptive learning technology into this study's framework to assure that children are well engaged to the activities given or the tasks assigned to them. Adaptive learning approach will be discussed further in the design section.

\subsubsection{Parent Role}

The parent-teacher relationship, the parents will be able to input certain real-life activities or situations into the system whereas the child can go through the system to be educated on those situations, this is to cope with home-life aspect. The parent role at home is mainly to complement 
the teacher role at school. Whereby the teacher concerns more on the educational materials based on curriculum and the parents concern is into the life routine situations.

\subsubsection{Technology Component}

$\underline{\text { Technology }}$ as an augmentation of human performance has been the core of this research for communication, contents and creativity as an attractive method to gain the autistics attention to the educational materials.

As discussed previously literature review chapter, this study aims to produce an interactive, web-based system to assess teachers and caregivers at schools or rehabilitation centres and parents at home as an alternative and complementary way of presenting the learning materials for children with autism.

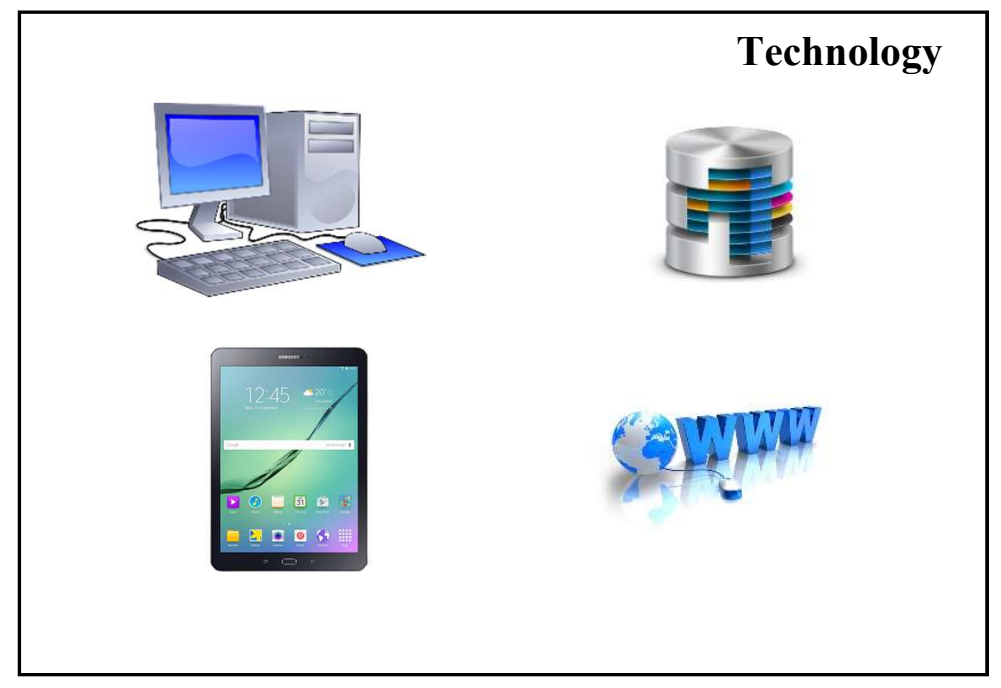

Figure 0.3: Technology component 


\subsubsection{A Web-Based System}

The Web is a term used to refer to the World Wide Web (WWW), or W3 (Vora \& Helander, 1997). Essentially, the Web is "a collection of protocols and standards used to access the information available on the Internet" (Vora \& Helander, 1997, p. 899).

There are three standards which assist the functioning of the Web: Uniform Resource Identifiers (URIs), which provide a mechanism for locating information, Hypertext Transfer Protocol (HTTP), which provides a mechanism for transferring information, and Hypertext Mark-up Language (HTML), which is a language for displaying information (Vora \& Helander, 1997).

It was demonstrated in the preliminary study chapter (section 2.3.4 and Section 2.3.5) that children with autism are comfortable and show excitement expressions using computers, as suggested by (Attwood, 2004; Bell, Cihak, \& Judge, 2010) computers offer many beneficial features and reliable results for individuals with autism and it has many advantages over the traditional rehabilitation methods (Epstein \& Klinkenberg, 2002; Evans \& Mathur, 2005; Fricker \& Schonlau, 2002; Gosling, Vazire, Srivastava, \& John, 2004; Umbach, 2004; Wilson \& Laskey, 2003).

Nonetheless, taking in consideration the other participants into the system such as the teacher at school and parents at home, it was anticipated that a web-based system would be feasible and facilitate the access from anywhere and anytime.

Main reasons for considering a web-based system are: Firstly, the knowledge and information to be taught to individuals with autism can be created via the system to demonstrate a real-life scenarios, schools subjects such as: English, Math or any other subject reflecting a range of 
activities that occur during the school day. Furthermore, adding interactivity feature to the system would make effective and efficient to grab the user's attention in order to complete the tasks. Secondly, the web-based system offers a potential platform to the teachers, caregivers or parents whereas they will be feeding the system with the educational materials. Moreover, they can manoeuvre the animations usage and the colour scheme to suite each child. Thirdly, autism centres operate daytime and children have to go home, therefore, there is a need for an application which can be accessed both at school and at home. Furthermore, an application to be easily updated and maintained frequently by the teachers or parents.

\subsubsection{Hypertext Mark-up Language}

As mentioned earlier, HTML is a language for displaying information on the Web. It does this by describing the logical structure of a hypertext document in such a way that it is understood by the web browser, which then processes the information and presents the document on the Web (Vora \& Helander, 1997). HTML is based on Standard Generalised Mark-up Language (SGML), an international standard "for defining mark-up languages. Authors mark up their documents by representing structural, presentational, and semantic information alongside content. HTML is one example of a mark-up language" (Khurana, 2001, p. 12). HTML can be created using a variety of tools. For example, a plain text editor such as Notepad can be used to write HTML from scratch, while more sophisticated authoring tools such as Microsoft FrontPage and Adobe Dreamweaver offer a 'What You See Is What You Get' (WYSIWYG) environment (Khurana, 2001).

\subsubsection{Hypertext}

There is no clear definition of the term 'hypertext' (Horton, 1990; Rada \& Diaper, 1991; Vora \& Helander, 1997). Typically, hypertext may refer to one or all of the following processes: 
information creation, information storage and management, and information presentation and access (Vora \& Helander, 1997). In order to understand the meaning of hypertext, a comparison to traditional text should be made (Nielsen \& Nielsen, 1995). Traditional text follows a sequential structure, with a linear sequence determining the order in which the reader looks at the text; hypertext on the other hand has a non-sequential structure, that is, there is no specified order in which the text should be read (Nielsen \& Nielsen, 1995).

Hypertext presents several different options to the readers, and the individual reader determines which of them to follow at the time of reading the text. This means that the author of the text has set up a number of alternatives for readers to explore rather than a single stream of information (Nielsen \& Nielsen, 1995, p. 2).

Hypertext applications have many beneficial features. For example, large collections of materials in varying media can be assembled and linked using hypertext (Vora \& Helander, 1997). In addition, hypertext facilitates the organisation of information in multiple ways, making crossreferencing of information easier and allowing multiple authors to combine information, promoting collaboration (Horton, 1990). These factors were considered relevant for this particular study where various forms of information were required to be organised and presented, and where several people might be responsible for authoring materials. Other benefits of hypertext include the fact that it can be controlled by the user and it has the potential for savings to be made on physical storage space and also on updating information (Nielsen \& Nielsen, 1995), features which were important considerations for this study. 


\subsubsection{An Interactive System}

It is commonly acknowledged that education at school consists of various activities which take place during the day, however those activities make the environment static in terms of the participants and the educational materials. Therefore, an interactive system is considered to fit in to illustrate and cop a dynamic environment which requires the individuals with autism to use daily with the assistance of the teachers and the parents in uploading different types of assessments.

A variety of different media formats are combined to present information and to facilitate communication between the user and the computer system. For example, interactive systems may include a mixture of graphics, text, sound and other media formats, which contribute to making the interaction an interesting and pleasant experience for the user. The aim of an interactive system for children with autism is essentially to gain their attention when communicating and using the system, moreover, applying the CCI design principles in the interactive system that implies an exciting involvement in the way the information is presented, retrieved and interacted with (Gillham \& Buckner, 1997). Nevertheless, to aid a user in accomplishing goals from some application domain. A domain defines an area of expertise and knowledge in some real-world activity (Patel, Yoskowitz, Arocha, \& Shortliffe, 2009).

In order for interaction and communication to take place there needs to be an interface to mediate between the user and the computer system; this is commonly referred to as the 'HumanComputer Interface' (Faulkner \& Faulkner, 1998). The interface is, therefore, an essential element in the interaction process, as it operates as a translator for both the user and the computer system. For a successful interaction to occur the interface must be able to facilitate an adequate translation. 


\subsubsection{Design Component}

Design The concept of design is the formation of learning resources in a systematic way with the employment of the design principles and the visual design in order to culminate the ultimate innovation image of the educational organisations.

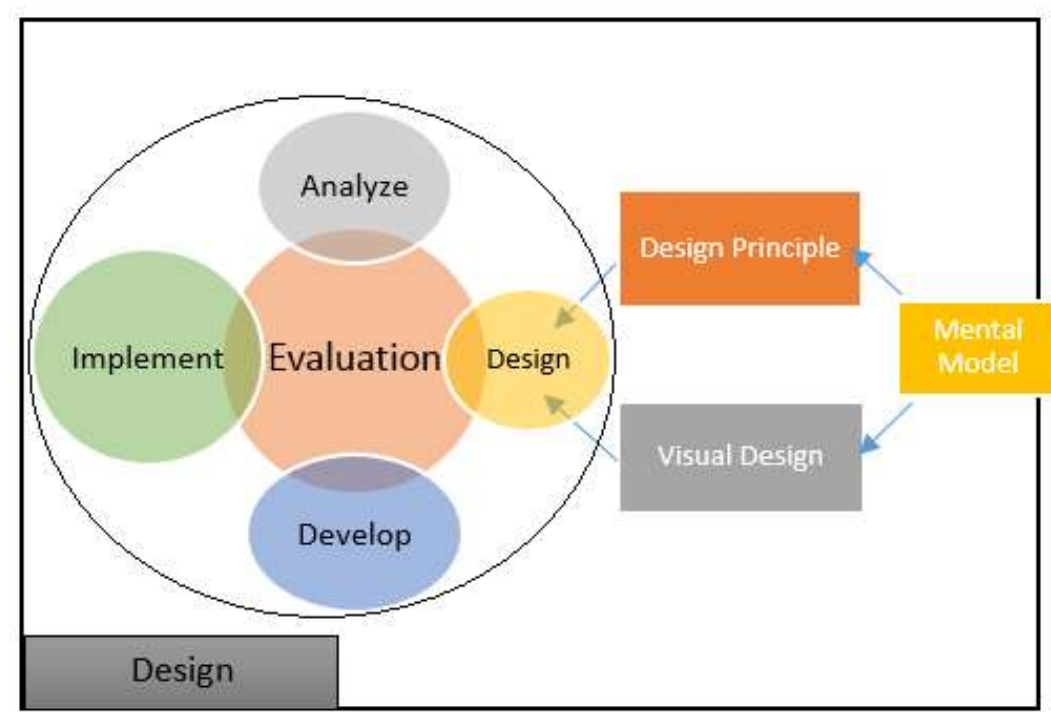

Figure 0.4: Design Component

\subsubsection{Instructional System Design Model}

Instructional System Design (ISD) model serves as a guide in developing ACMLS framework. There are different approaches to ISD but all have the basic concept of ADDIE, which is the acronym for the five phases as shown in figure 4.3 (Analysis, Design, Development, Implementation and Evaluation) as a systematic approach of designing an instructional system. Each of the components in this approach has its relevance in the design process. The brief description of each phase is given below.

Analysis: This phase comes first in the systematic approach in developing an instructional system. The major activity in this phase involves creating a user profile for the instructional system 
and understanding the general requirements of how to achieve the learning objective. A detailed analysis was discussed in chapter 1, chapter 2 and chapter 3.

Design: This phase deals with the presentation of the learning objectives, contents, subjects, assessments, exercise and quizzes in order to achieve the aim and goal of this study which is provide children with autism with an alternative learning system instead of the traditional methods and provide caregivers with a gateway manage the materials given to the children as well as an administration privileges to monitor learners progress. This phase is covered in chapter 5 .

Development: This phase focuses on the creation and the content assets assembly which is described in the design phase until the production of the application as an outcome of the analysis and design phases. This phase is covered in chapter 6 .

Implementation: This phase involves the experimental stage where the developed system is used by the targeted users. Chapter 7 introduced the implementation phase as part of the evaluation.

Evaluation: This phase checks the effectiveness of the developed system by taking certain measurements and feedbacks. This phase is covered in chapter 7.

\subsubsection{Design Principles}

Michaud, Duquette, and Nadeau (2003) found that it's so important to adhere the design principles which take the key characteristics of autism character in consideration while the system or the framework development.

Design principles to consider in designing ACMLS framework such as: 
- Images and photos are a more effective way of representing an object or event than words for children with autism. In addition, the device should use simplified short messages (three words or less) which are frequently repeated to support learning. Messages should also refer to concrete things rather than abstract thoughts and concepts.

- The design of ACMLS framework should take in consideration the individual differences of the children with autism who are going to use it, each individual has certain needs and requirements which should be addressed and taken care of so to be a successful intervention.

- The inclusion of parents input into the ACMLS framework is to use it at home, which will help in intensifying the intervention for the children. Parents will be given the accessibility to add and upload educational materials to the system.

- The colour scheme of the system shouldn't be anxious to children. According to (Speaks, 2011) children with autism may show sensitivity to certain colours. Therefore, a control panel will be designed for parents and teachers in order to customise the front-end system to the liking of the child.

\subsubsection{Visual Design}

As mentioned earlier the use of multimedia in learning involve of the usage of text, images, animation and audio as a medium of knowledge transfer to learners. Multimedia can be referred to the text representation in a written, audio or animation and videos (Mayer, 2005) which can be referred to the mental model or construction form from a given multimedia representation (words, audio, pictures and animations). Multimedia elements to be considered when constructing or building a multimedia learning medium (ACMLS) for teaching children with autism involves of: 
- Symbols can be used as an alternative representation to text (Leach, 2010), although it is important that there are concrete representations due to children with autism struggling with understanding abstract concepts.

- Children with autism can have problems understanding abstract concepts; so abstract metaphors should be avoided (Mesibov, Thomas, Chapman, \& Schopler, 2007), graphical representations should be as realistic as possible (Leach, 2010).

- Children with autism can exhibit even greater difficulties with reading and so text should be kept to a minimum (Leach, 2010) and their strength for visual processing incorporated within the design wherever possible (Barry \& Pitt, 2006; M. Davis, Dautenhahn, Powell, \& Nehaniv, 2010; Van Rijn \& Stappers, 2008).

- The use of certain sounds can trigger anxiety in some children with ASD who have auditory sensitivities and so should be used with caution (M. Davis et al., 2010; Leach, 2010; Putnam \& Chong, 2008), ensuring there is an option to turn sound on and off.

- Colour schemes should be kept simple for children with autism, with the option to change colours, for example the background colour to reduce contrast with text and aid reading (Leach, 2010).

- As mentioned earlier it is important to consider sensory issues when using animation, sound and highlighting through different colours when designing for children with autism (M. Davis et al., 2010; Leach, 2010; Putnam \& Chong, 2008), particularly if being used in conjunction with one another as they can struggle to know where to direct their focus (M. Davis et al., 2010). 


\subsubsection{Mental Model}

A user generally has certain expectations about the behaviour of a computer system and this is typically referred to as the user's mental model (Allen, 1997; Faulkner \& Faulkner, 1998). This type of model exists in a person's head and so obviously it is not possible to see such a model (Allen, 1997). People form their mental models of systems through experience and training (Norman, 2014). In complex situations people tend to make inferences based on prior experience, developing mental models dynamically to help them cope with a new or difficult situation (Preece \& Rombach, 1994). Therefore, “the user's mental model of an information system has a critical impact on the user's ability to use systems effectively" (Chen \& Sharma, 2002, p. 51). Unfortunately, mental models formed by users are frequently vague and incomplete and it is, therefore, important that the interface of a computer system is designed in such a way that it facilitates the attainment of an accurate model of the system by the user (Faulkner \& Faulkner, 1998; Preece \& Rombach, 1994). A means of assisting a system designer to demonstrate a potential system and facilitate discussion of a user's mental model is through development of conceptual models.

\subsubsection{Education Component}

$\underline{\text { Education }}$ The concept of education is here concerned with the aims, objectives \& values, the organisations, processes $\&$ culture of the instruments $\&$ institutions that society has formulated to address the its needs to educate lifelong learners. 


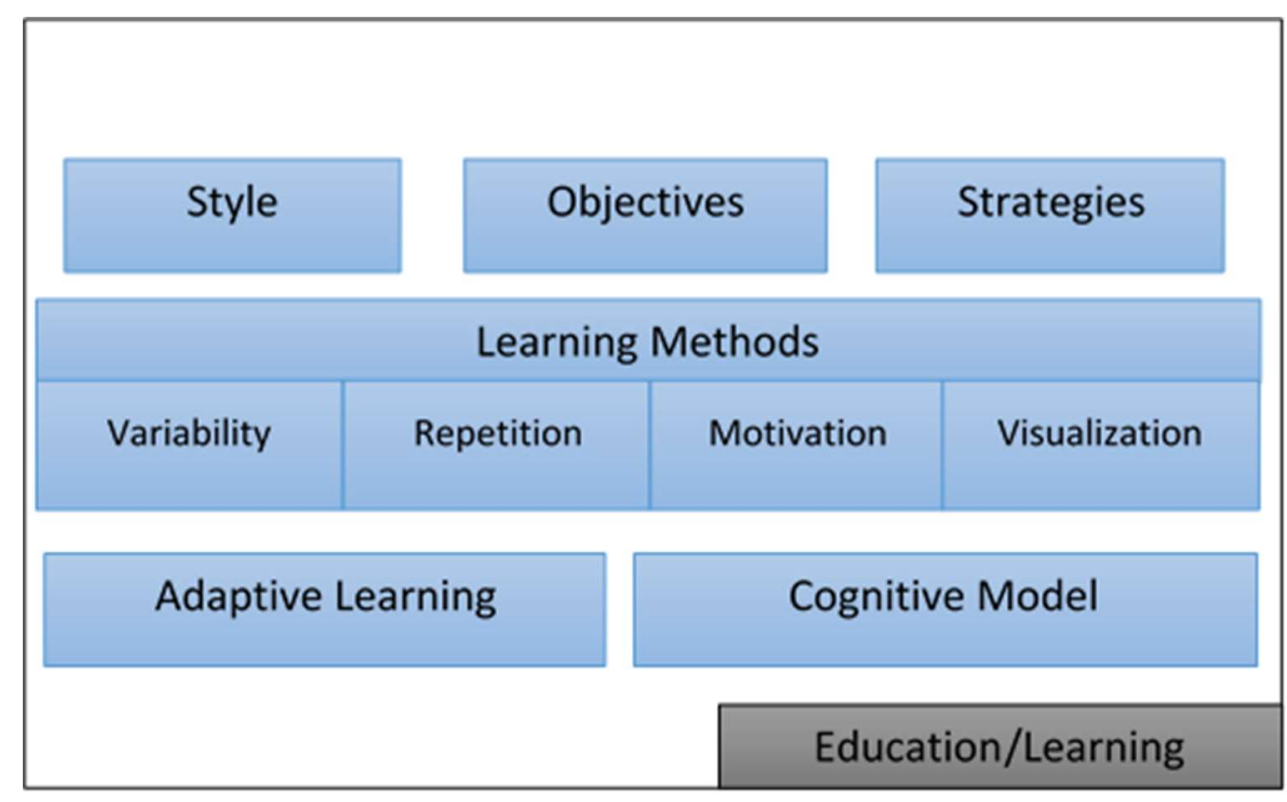

Figure 0.5: Education Component

\subsubsection{Learning Objectives}

Defining the learning in objectives helps mainly in the learning system design and to keep the focus of the learning system framework main goal so that don't deviate from the main objective. And as a learning major objective is set to educate children with autism and get the fullest attention towards the educational materials presented to them at school by the teacher or caregivers and at home by the parents. And in order to do that; the CCI design principles have to be present in the learning system framework design process.

As mentioned earlier in the literature review chapter that children with autism have a very low cognitive skills compared to typical children. Therefore, children with autism require a simple visual presentation of what to be learnt by using pictures for example to represent an action or information which needed to be delivered to children instead of using an abstracted text presentation. Picture Exchange Communication System (PECS) as an example of how the information or actions been presented to children with autism. 
As mentioned earlier that the main objective of this research is to build a useful tool for children with autism as a learning tool which fed by the teacher at school or parents at home with educational materials, while the system design will ensure the children are paying full attention to learning materials. In addition to that learning presentation style is a main factor that influences the children attention towards the materials.

\subsubsection{Learning Style}

Learning style is the component that assists in the achievement of the learning objective, it is considered the main factor that can influence the learner interests and progress. And due to many different learning styles available according to S. E. Davis (2007) such as: Visual, Aural, Verbal, Physical, Logical, Social and Solitary. The variability of the learning styles adds effectiveness to the learning tools so it cope those different styles to keep the children level of attention as high as possible to the learning materials. None of the mentioned learning styles earlier considered as the best style to be followed to educate children with autism due to the individual differences among them. Hence, it would rather depend on what is applicable to each learner which might not be suitable to another.

The representational style is considered in this research to present the educational materials or contents with a simple picture for example using ball picture to represent "I want to play" while other contents have to be presented in a decorated textual form. Audio is considered as add-on feature to the textual forms of presentation. This indeed required when it comes to learning Quran verses (Banire, Jomhari, \& Ahmad, 2015). And as mentioned earlier the objective of this research is to come out with ACMLS framework for children with autism which can help in teaching vary number of topics with the help of the teachers or parents. 
Contents presentational style is the method of presenting the contents to children, and as illustrated in the literature review chapter the different interests of the children with autism and their cognition abilities. Therefore, different presentational styles are considered for educational material such as:

\section{Video Presentational Style}

Video presentational style could be referred to as a live scene of the context of a message or content to be taught. This visual style is considered in this study in order to facilitate comprehension in learning. The video presentational style is adopted from video modelling technique where it is used for content illustration in learning for children with autism which supports their needs for real scenarios (provide them with selective attention) in certain elements in the visual display (McCoy \& Hermansen, 2007). The use of video modelling in children with autism has been effective in relaying a message to them.

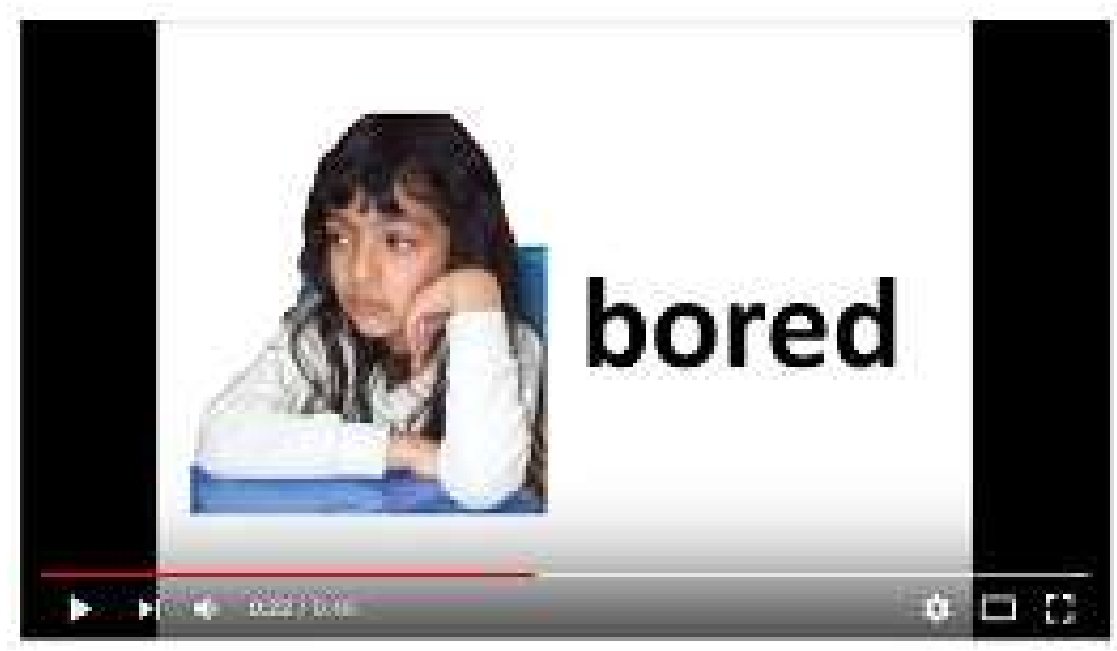

Figure 0.6: Video modelling 
Video modelling as showed in figure 4.5, it involves the real life visual display to the targeted learner without any interruption while watching (Sigafoos et al., 2007). The types includes: include Video Self Modelling (the use of the learner's image as element to get attention) (Robson, Blampied, \& Walker, 2015; Whitlow \& Buggey, 2003), Scene view video modelling (the use of scenario or scene of an event) (Moore et al., 2013) and Point-of-view video by (Hine \& Wolery, 2006; Tetreault \& Lerman, 2010), to explain the how to prepare an afternoon snack to a 4 year old child with autism. This process involves the use of exact action that is to be done by the learner in sequential steps. This method was effective in training this child, although he was sometimes guided by prompts. All of these types of video modelling are effective as a means of intervention in children with autism. However, the use of Scene view video modelling could be used as a general representation of an event. This kind of video modelling does not represent the whole information but part provides part of the information that can represent the whole information. Hence, Scene view video was adopted as one of the Learning Method.

\section{Transitional Image}

The use of static image involves the use of picture that could be used to further illustrate a text or action. According to Morrison, Ross, Kemp, and Kalman (2010) the use of a picture or animation was identified as the best way to facilitate learning in children. There has always been the debate on which is better when animation and static images are used in teaching children with autism. There are some studies that support the use of static picture as the best way to enhance learning. Static picture keeps the information to be conveyed permanently where the learner can 
always refer back to the message unlike the animation that transits and does not leave the image or scene for a long period of time in order to be mastered before it disappears (Hegarty, 2004).

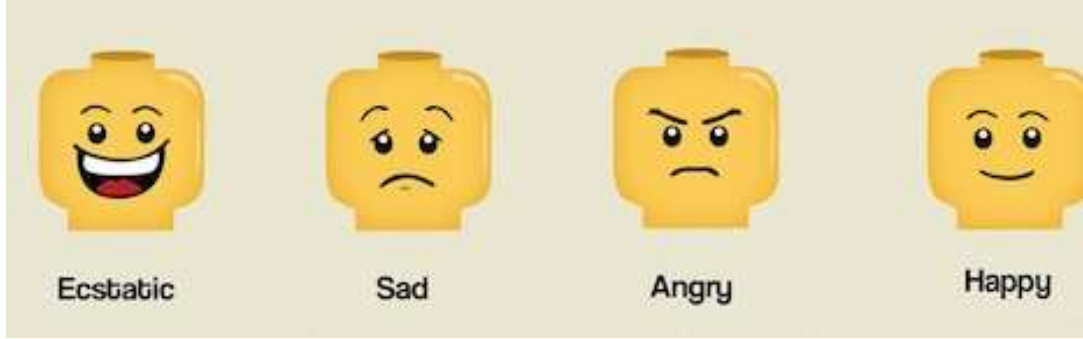

Figure 0.7: Static picture presentation

However, the use of static picture as seen in figure 4.6 could be a best way of communicating message in learning but this does not go well with children with autism because they require stimulating effects to keep their attention. Static pictures appear boring to them that they give little or no attention to it.

According to one of the teacher, he said: The use of static pictures in a transitional manner attracts the attention of children with autism more rather than static alone.

More so, it has been observed in the preliminary study that some children with autism are obsessed with a rolling fan they also very attentive to systemizing toys such as train (Golan et al., 2010).

\section{Humanoid Animations Style}

Animation and colourful images have the features to attract the attention of learners especially children with autism (Doyle \& Arnedillo-Sánchez, 2011). The findings showed that 90\% of the learner with autism benefited from this mode of presentation as compared to other modes such as video, image and audio. It is quiet easy for learners to put the content of the lesson into memory 
because of its illustration effect. However it may require high processing memory (cognitive skills) for some learners with autism. Hence, this mode of presentation might need to be extended in order to cater for other learners.

\section{Decorative Style}

The decorative animation simple means abstract visual representation of the learning content. It does not present the visual meaning of the learning content but provides a stimulating effect as used in sensory room for visual stimulation. It consists of only one method of illustration which is abstract animation.

\section{Sad Happy Angry Surprised}

Figure 0.8: Abstracted decorative style

The abstract dynamic image is referred to as the animation of multiple colours as seen sensory room. This stimulus has been effective as a visual stimulation for children with autism as it calms them and gives them satisfaction. This dynamic multiple colours can be perceived by these children as visual stimulations that attract their attention. Since there is no information to be processed from this visual, the need for cognitive ability will be less required. This effectively makes the children focus on the screen that has the displayed colourful text (see figure 4.7) due to the high perceptual load which is responsible for reduction of cognitive load. This is in line with load of theory and attention as well as cognitive control (Lavie, 2010). 


\subsubsection{Learning Strategies}

The learning strategies of the ACMLS framework are focusing on how to achieve the learning objective which was set, and throughout the learning styles. Morrison et al. (2010) suggested that those strategies should be followed in an instructional design. These strategies are:

1. Colourful pictures: children with autism showed an attraction to colourful pictures and objects. This will help them to pay more attention.

2. Simple illustration: teaching contents with simple illustration helps children with low cognitive skills.

3. Contents repetition: as one of the children with autism characteristics or rather strength is they have the repetitive behaviour or action, therefore, they like the repetitive actions which help them to pay attention to the given tasks.

4. Animations: children with autism are attracted to the animation characters, it helps too in increasing their attention level.

5. Short lessons: children with autism have anxious to the long duration of a learning sessions. Therefore, a short lesion duration is a good method to be followed in order to achieve the learning objective.

6. Clear and colourful font: this helps the children to read clearly and noticeably, and by making the text colourful that makes pay attention to the given learning text.

\subsubsection{Learning Methods}

Learning methods are the various ways the learning objective can be reinforced in order to achieve the goals. Four types of methods are proposed after interviewing teachers, parents and 
from observations during the preliminary study. These methods are: Variability, Repetition, Motivation, and Visualization.

\section{(a) Variability-progress}

Variability is a key component in children's learning and development. Variability is suggested for better acquisition in children with autism learning, by accumulation of instances of particular words, information presentation and situations structure. This should assist children cognitive skills ability growth for instance: by presenting a certain situation in diverse ways to children should provide a more complete sample of possible forms of reactions. Another example is language presentation to children in different ways and forms provides a larger sample of possible expressions when used to form a sentence

\section{(b) Repetition - Memory}

Reinforcement should be encouraged in any learning application for learners with autism as it enhances their learning. Reinforcement also aids the memory in adapting to the content better. There are two principles that can be used to aid incorporation of content especially when the learners are slow in learning or the content is strange to them. The principles are principle of organisation and principle of repetition (Alessi \& Trollip, 2001). The rule of repetition is considered as one of the learning strategies because one of the strengths of children with autism is rote memory which supports repetition (Quill, 1997). They like to repeat things and are intent by repetition. In addition, repetition was one the ways the speech therapist uses in emphasizing the learning content to children with autism in mostly all autism centres or schools. The principle of organisation also plays a good role in presenting the content. The content should be presented in 
such a way that the children are motivated with the first look based on the design principles and learning strategies which mentioned earlier.

Repetitive pattern of the content will enhance their attention and ability to retain the content. Repetition could also be considered as a way of making perception easier for learners (Alessi \& Trollip, 2001). Learners can be persuaded to repeat content of the module if repetition is used as part of the presentation and it aids learner in eventually mastering the content.

\section{(c) Motivation - Reward}

Motivation in learning is imperative as identified by psychologist especially for learners with autism who always try to avoid learning or pay less attention. Rewarding learners always gives them the feel of progressive achievement. It has been chosen as a way of increasing their attention and attracting them to the learning topics. This perspective also make the learning interesting (Hulusic \& Pistoljevic, 2012).

\section{(d) Visualisation - Presentation}

Visualisation as mentioned in the learning guidelines must be followed for effective learning outcome especially when children are the main targeted learners and based on their characteristics. Learning guidelines motivates and attracts the attention of the children during learning session for example plain text is usually ignored by average learners, therefore, in order to gain attention for those children with autism especially those with attention impairments, visual modes of presentation such as animated images and audio is used. This study made use of colour code as a way of animating the text and overall system appearance in order to fit the learners according to their profile such that the colour of the text changes as its being read in order to gain the most of the attention of learners to the educational contents. 


\subsubsection{Adaptive Learning Approach}

Adaptive learning approach is an educational approach, it uses the computers as interactive learning medium, and manages learners according to their unique needs. Computers as a learning medium adapts the presentation of the educational material and curriculum to help students according to their intellectual capabilities (Battou, 2017; Ibrahim, AlSharabi, Djemal, \& Alsuwailem, 2016).

Nonetheless, adaptive learning method attempts to transform the students from passive receptor of knowledge and information to a collaborator in the educational activity. It also provides valuable information and feedback to teachers and parents as one of its key features in order to use it in a more constructive way.

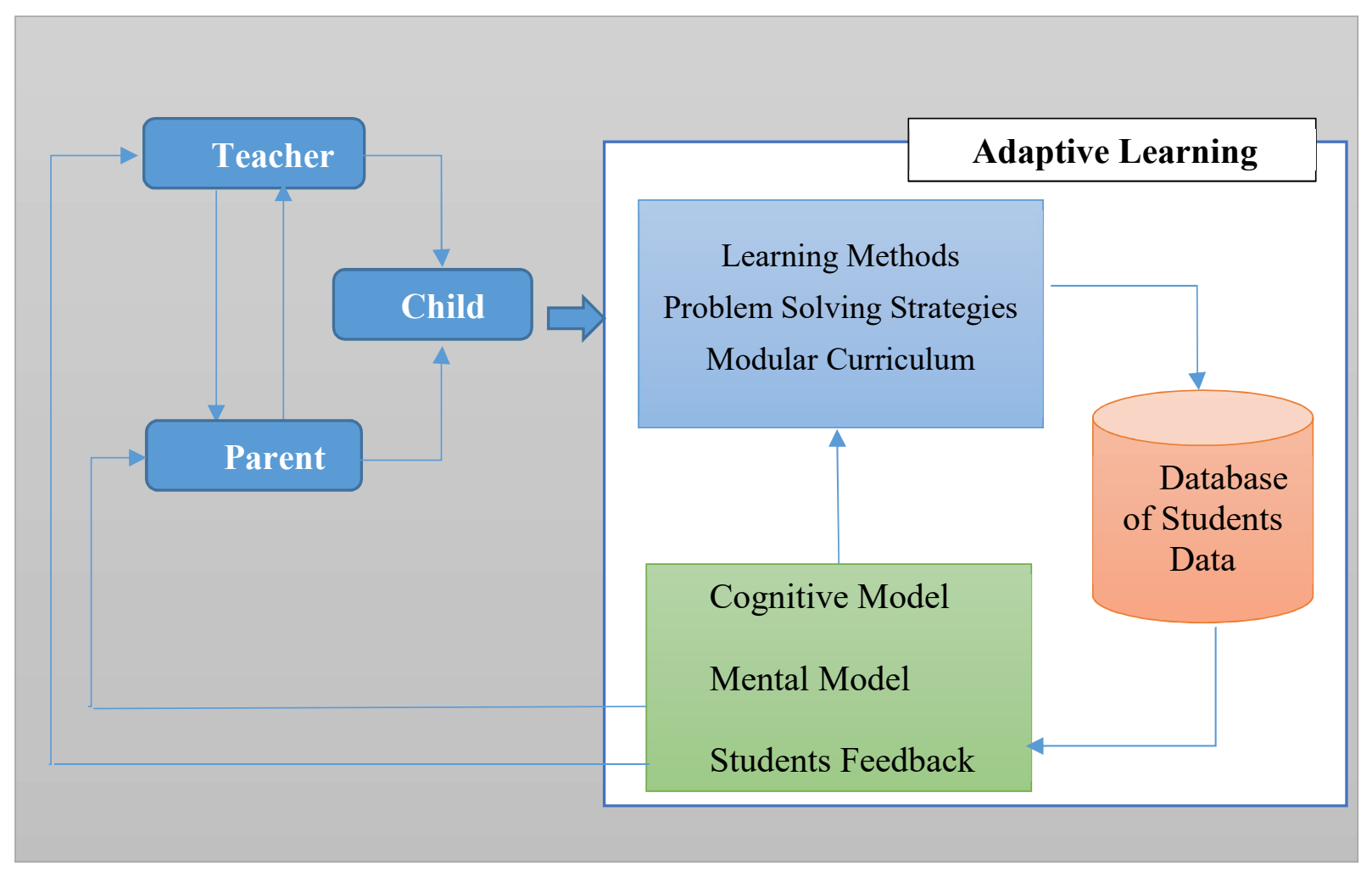

Figure 0.9: Adaptive Learning Flow 
Kerns (2013) listed many benefits of using adaptive learning technology such as:

Can be customised to Fit All Learning Levels Adaptive learning can be personalized learning system which adapts to all learning capabilities. The teacher needs to set the parameters for each individual for the first time and save it as part of the learner profile. When the learner starts using the adaptive technology approach, it will detect what difficulties or hardship the learner is facing and store it in the database as a row data and send a feedback to teacher as part of the learner progress.

The learning style used in this approach is flexible style whereby the learner can use different strategies to solve the presented problems such as textual, verbal, and visual or animation presentation. So that learners be able to achieve the learning objectives and pay closer attention to the given tasks and materials.

Provides Real-time Feedback Adaptive learning technology is designed to: Firstly, collect learner immediate response or answer to the presented problems. Secondly, learner while answering will prompted with an indication of whether the answer is correct or wrong. Lastly, send the learner's answers to the parents or teacher as a feedback and part of the learner progress.

By keeping track of each learner, teacher or parents will have a better vision for a future tasks to be given for those who're facing difficulties getting past one lesson or a task.

Accelerates Students' Progress As mentioned before in the literature review chapter, children with autism are varying in skills impairments and learning pace. Therefore, it becomes so difficult to design a 'one size fits all' concept. However, adaptive learning technology helps to design educational materials based on the CCI concepts in section 3.7 aiming to gain the learners attention 
and faster progress. This way, every learner benefits from the assessment equally and no one is left behind.

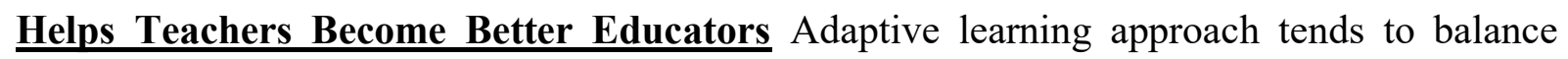
between the face-to-face instructions and the online instructions. It doesn't mean adaptive learning meant to replace the educators, it just help to initiate a self-paced guidance system and build up the independence aspect into the learners.

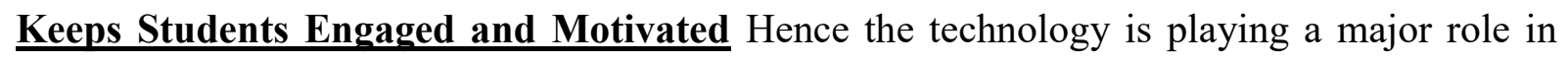
education nowadays, as well as the children with autism are showing an interest in technology. Therefore, the adaptive learning approach is a motivation factor in the learner's life. Consequently, children can become more engaged with the educational materials or the tasks assigned to them via the system in order to achieve it aiming to a better scores and understanding.

\subsubsection{Cognitive Model}

Literature has shown that children with autism have low cognitive ability and information processing skills as compared to typical children, they have difficulties in interpreting or understanding the thoughts and feelings of other people, may have difficulty with empathizing and often feel confused by other people's behaviour (Baron-Cohen, 2000; Ozonoff \& Miller, 1995). Hence, they require simple visual illustration for information processing by using a picture to present an action in information given rather than representing the whole information for example the PECS. Children with autism prefer the visual presentations with less information details which empower the learner's state of mind to absorb the information and lead to a faster information processing rather than detailed visuals which may confuse them and disturb their behaviour. 


\subsubsection{Multimedia Strategy and Perception}

The way and manner learners perceive and attend to information are key factors in achieving a meaningful learning. However, attention of children with autism is not easily achieved. Thus, there are different principles to be followed for different components such as text, picture, audio and background of the presentation style (Alessi \& Trollip, 2001). The principles are:

a. Information should be simple and easy for perception.

b. Dynamic approach should be adopted in order to attract and sustain attention in learning.

c. The position of the relevant content should be conspicuous.

In order to enhance the learner's focus and attention, the font size and colour should be considered, the audio should be clear and audible and the picture should be clear and attractive.

According to Wise (2014) listed other principles to be considered in addition to the above list:

1. Predictable action: system designers are advised to let the child know what will happen next. For example, "After you finish the dinner, it is time to brush your teeth", or "In five minutes it is time to turn off the computer and start your writing assignment". And in order to selfmonitoring a timer should be placed within the application or the activity such as a countdown timer. So when they are given a task or assignment they do better if they know when the task will end.

2. Avoid loud sounds: many children with autism are audio hypersensitive so it's important to avoid loud sounds. 
3. Set expectations, be consistent, and follow through, by making a routine activities. Children with autism have the challenging behaviours so in order to avoid that action should be predictable. An example of predictability would be having a set routine each night.

4. Acknowledge the child or students for complying such as praising or rewarding when complying with the rules.

5. Tell the child specifically what you expect and allow him to earn privileges for complying with your expectations

6. Give Choices, children in general like to feel a sense of control over their world. Hence, we are dealing with children with autism in this study. Therefore, limiting the choices to two to four options is beneficial, as may get overwhelmed with too many choices and cannot decide.

\subsubsection{Problem Solving Strategies}

This form of knowledge is the basis of analysis and creativity and may involve the application of mental models. I argue that the key capabilities are those of recognition, open-mindedness, backtracking and re-formulation. My own work in this area resulted in a published paper to identify the steps that the learner would need to undertake to formulate computer models (Millwood \& Stevens, 1990), based on the experience gained in formulating the Modus project to design modelling software:

- Identifying a purpose;

- Having concern for presentation and communication;

- Constructing an interactive simulation;

- Picturing the end-product;

- Identifying elements; 
- Characterizing elements;

- Identifying relationships and

- Characterizing relationships.

Even in the higher, informal and professional sectors of education, complexity of education is matched by complexity of learning outcomes which may include:

- Skills development;

- Knowledge acquisition;

- Improvement in strategic, analytic and creative capacities;

- Attainment of competence;

- Establishment of attitudes and values.

Each of these societal purposes and these learning outcomes demand different approaches and understandings for the theorist and may develop at varying rates or found to be diverse in relation to context, location and culture (Felder \& Brent, 2005).

\subsection{ACMLS Framework Architecture}

Figure 5.10 shows that ACMLS framework adopts Client-Server architecture which describes the system structure in which a client requests an action from the server. And in return, the server replied to the client's request with a relevant actions. The resources in the server side are considered as a learning resources, which can be accessed from multiple and different clients by teacher, parents or learners.

Figure 5.10 shows the requests and responses between client and ACMLS server. The process starts from mobile client by requesting certain action over the internet medium over the TCP/IP 
protocol and once the request arrives to the ACMLS server, the server processes the request and extracts the information from the database, then replies to the client with the learning materials and educational resources in a form of HTML format. The client side receives the HTML file ad render it in the internet browser. This process will be repeated until the learning session for this specific user is over. All users' actions, results and history will be stored in the database as part of the users' progress for future analysis.

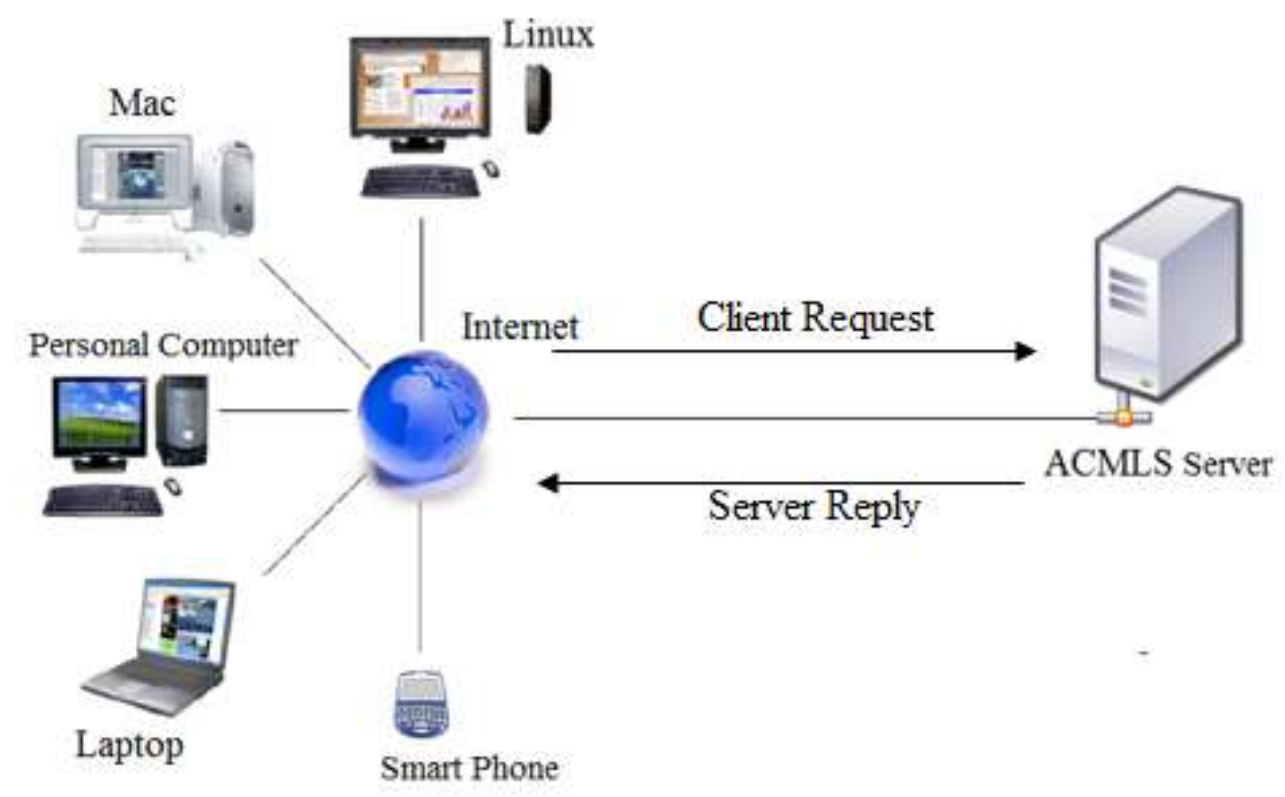

Figure 0.10: ACMLS Client-Server Architecture

The ACMLS as mentioned earlier is a client-server system and it emphasizes a dynamic structure for learning rather than a fixed or static structure. By adapting learning contents and activities to the learners and their abilities, the system is able to prepare a suitable and personalized learning platform model for learners as per their user profile. The system structure consists of three main parts: (1) Client with a browser support such as: laptop, tablet, personal computer or smart phones. (2) Server. (3) Teacher or parent portal. The client can be any touch-screen device which 
is connected to server. The server is a standalone device that manages, personalizes and provides the client with the learning materials and educational resources. Teacher or parent portal is a gateway for uploading the learning materials and activities as well as the educational resources, more so monitors the learner's behaviour and progress statistics.

The data transmission speed within the client-server system depends on different factors such as devices processor, connectivity and data type of the learning materials.

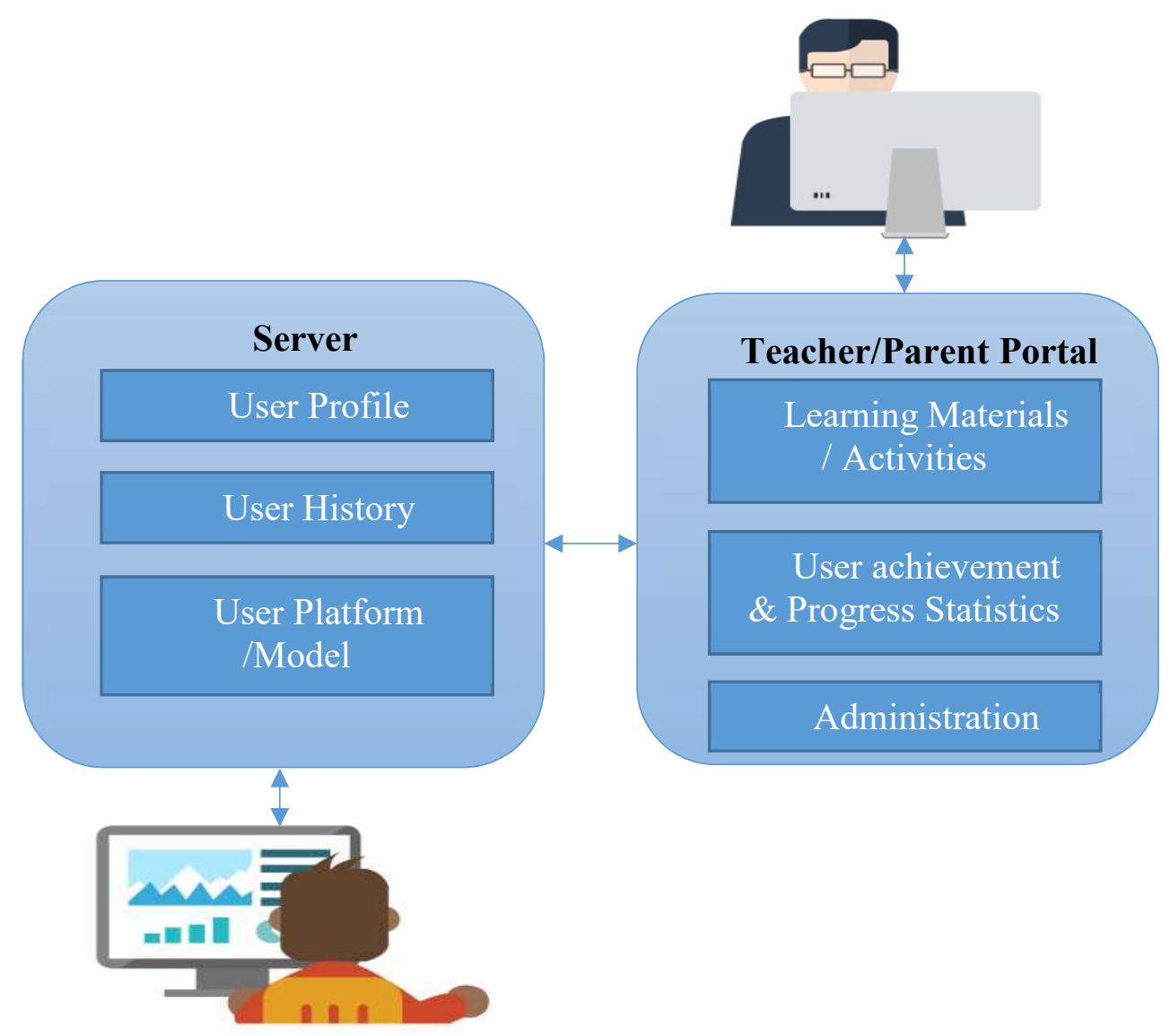

Figure 0.11: ACMLS Architecture

Figure 5.11 shows the ACMLS architecture; the learning process in ACMLS framework starts with the client requests to login and the user platform is personalized based on the user profile. A 
user profile which fed to the system with the help of the teacher or parents using analytical games, questionnaires and interactive games such as PECS, this to assess to personalize the user's platform. During the learning process, the results of quizzes, behaviour and interactions are processed by the server and saved in the database for progress monitoring, analysis and user's development evaluation. On the other hand the teacher or the parent login to the system via administrative portal, they will be able to upload, create and administer the learning materials and resources. Teacher or parents can generate statistics, user history and progress analysis.

\subsection{ACMLS Framework Learning Process}

Learning process as figure 5.12 shows, it starts with teacher or the parent by identifying the learning objectives and what to be taught to the learners, identifying the learning objectives shall make the learning process a goal oriented rather than deviating away from the main objective for starting this process with learners. Teacher or parent have to set the learning process objectives which has to be achieved, and the learning styles as well as the learning strategies used to accomplish the objectives. Another important requirement is the learning methods which should be applied too so that the learner attention doesn't drop and deviate taking in considerations that the targeted users are children with low cognitive abilities as compared to typical children. Therefore, they require a simplified visual illustration of the learning materials.

Learning process is an action that has to be accepted by the targeted learners, they should be self-determined and willing to go through this process. The teacher or parents should observe the learners in order to build the learning environment which aims to empower learners and motivate them in the initial stages so that they can lead to understanding the learning process and tasks before they independently use the system. Assessments and quizzes are given to the children with 
autism in order to gauge their learning progress. The system provides a motivational feedback to the learner's interaction so that can induce the learner attention to the given tasks.

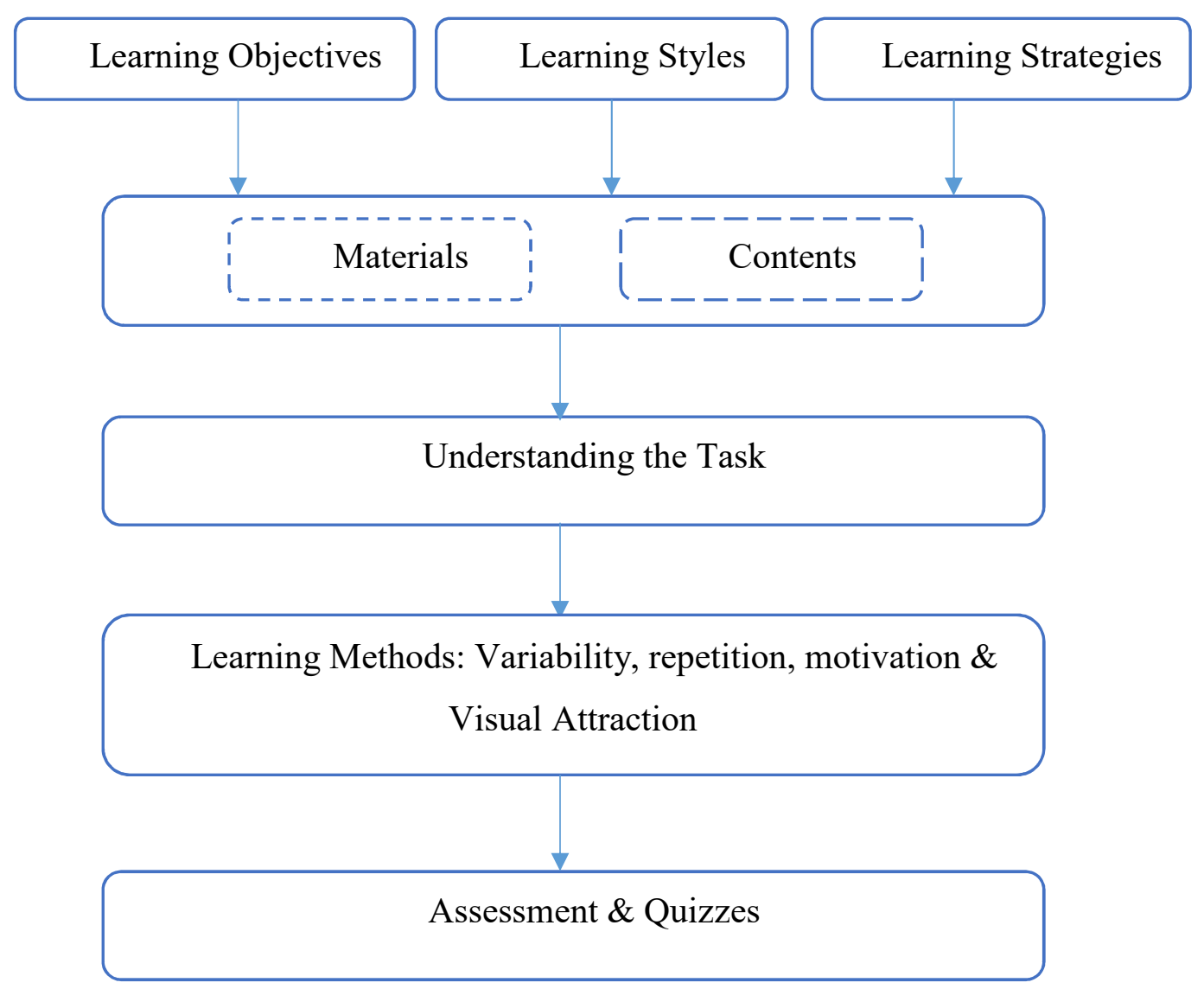

Figure 0.12: The ACMLS learning process

\subsection{Conclusion}

This chapter presented the proposed ACMLS framework and its four components: education, design, participants and technology, each component was explained in details.

Participants' component was focused on the main participants (teachers, parents and children with autism) and role played for each one of them in ACMLS usage. 
Technology component illustrated the tools used to develop the prototype and the use of HCI tools which will be incorporated into the ACMLS framework in order to provide an interactive system for the system participants.

\section{References:}

Alessi, S. M., \& Trollip, S. R. (2001). Multimedia for learning: methods and development: Allyn \& Bacon, Incorporated.

Allen, R. B. (1997). Mental models and user models Handbook of Human-Computer Interaction (Second Edition) (pp. 49-63): Elsevier.

Attwood, T. (2004). Cognitive behaviour therapy for children and adults with Asperger's syndrome. Behaviour Change, 21(3), 147-161.

Banire, B., Jomhari, N., \& Ahmad, R. (2015). Visual Hybrid Development Learning System (VHDLS) framework for children with autism. Journal of autism and developmental disorders, 45(10), 3069-3084.

Baron-Cohen, S. (2000). Theory of mind and autism: A review. International review of research in mental retardation, 23, 169-184.

Barry, M., \& Pitt, I. (2006). Interaction design: a multidimensional approach for learners with autism. Paper presented at the Proceedings of the 2006 conference on Interaction design and children.

Battou, A. (2017). Designing an Adaptive Learning System Based on a Balanced Combination of Agile Learner Design and Learner Centered Approach. American Scientific Research Journal for Engineering, Technology, and Sciences (ASRJETS), 37(1), 178-186.

Bell, S. M., Cihak, D. F., \& Judge, S. (2010). A Preliminary Study: Do Alternative Certification Route Programs Develop the Necessary Skills and Knowledge in Assistive Technology? International Journal of Special Education, 25(3), 110-118.

Chen, Q., \& Sharma, V. (2002). Human factors in interface design: an analytical survey and perspective Human factors in information systems (pp. 45-54): IGI Global.

Davis, M., Dautenhahn, K., Powell, S., \& Nehaniv, C. (2010). Guidelines for researchers and practitioners designing software and software trials for children with autism. Journal of Assistive Technologies, 4(1), 38-48. 
Davis, S. E. (2007). Learning styles and memory. Institute for Learning Styles Journal, 1(1), 4651.

Doyle, T., \& Arnedillo-Sánchez, I. (2011). Using multimedia to reveal the hidden code of everyday behaviour to children with autistic spectrum disorders (ASDs). Computers \& Education, 56(2), 357-369.

Epstein, J., \& Klinkenberg, W. D. (2002). Collecting data via the Internet: The development and deployment of a web-based survey. Journal of Technology in Human Services, 19(2-3), 33-47.

Evans, J. R., \& Mathur, A. (2005). The value of online surveys. Internet research, 15(2), 195-219.

Faulkner, C., \& Faulkner, C. (1998). The essence of human-computer interaction: Prentice Hall London.

Felder, R. M., \& Brent, R. (2005). Understanding student differences. Journal of engineering education, 94(1), 57-72.

Fricker, R. D., \& Schonlau, M. (2002). Advantages and disadvantages of Internet research surveys: Evidence from the literature. Field methods, 14(4), 347-367.

Gillham, M., \& Buckner, K. (1997). User Evaluation of Hypermedia Encyclopedias. Journal of Educational Multimedia and Hypermedia, 6(1), 77-90.

Golan, O., Ashwin, E., Granader, Y., McClintock, S., Day, K., Leggett, V., \& Baron-Cohen, S. (2010). Enhancing emotion recognition in children with autism spectrum conditions: An intervention using animated vehicles with real emotional faces. Journal of autism and developmental disorders, 40(3), 269-279.

Gosling, S. D., Vazire, S., Srivastava, S., \& John, O. P. (2004). Should we trust web-based studies? A comparative analysis of six preconceptions about internet questionnaires. American Psychologist, 59(2), 93.

Hegarty, M. (2004). Dynamic visualizations and learning: Getting to the difficult questions. Learning and Instruction, 14(3), 343-351.

Hine, J. F., \& Wolery, M. (2006). Using point-of-view video modeling to teach play to preschoolers with autism. Topics in Early Childhood Special Education, 26(2), 83-93.

Horton, W. K. (1990). Designing and writing online documentation: help files to hypertext: John Wiley \& Sons, Inc.

Hulusic, V., \& Pistoljevic, N. (2012). "LeFCA": Learning framework for children with autism. Procedia Computer Science, 15, 4-16. 
Ibrahim, S., AlSharabi, K., Djemal, R., \& Alsuwailem, A. (2016). An adaptive learning approach for EEG-based computer aided diagnosis of epilepsy. Paper presented at the Intelligent Technology and Its Applications (ISITIA), 2016 International Seminar on.

Kerns, D. (2013). Six key benefits of adaptive learning.

Khurana, R. (2001). Html 4 U: APH Publishing Corporations.

Lavie, N. (2010). Attention, distraction, and cognitive control under load. Current Directions in Psychological Science, 19(3), 143-148.

Leach, C. (2010). The use of Smartboards and bespoke software to develop and deliver an inclusive, individual and interactive learning curriculum for students with ASD. Journal of Assistive Technologies, 4(1), 54-57.

Mayer, R. E. (2005). The Cambridge handbook of multimedia learning: Cambridge university press.

McCoy, K., \& Hermansen, E. (2007). Video modeling for individuals with autism: A review of model types and effects. Education and treatment of children, 183-213.

Mesibov, G., Thomas, J. B., Chapman, S., \& Schopler, E. (2007). TEACCH Transition Assessment Profile: TTAP: Pro-ed Austin, TX.

Michaud, F., Duquette, A., \& Nadeau, I. (2003). Characteristics of mobile robotic toys for children with pervasive developmental disorders. Paper presented at the Systems, Man and Cybernetics, 2003. IEEE International Conference on.

Millwood, R., \& Stevens, M. (1990). What is the modelling curriculum? Computer Assisted Learning (pp. 249-254): Elsevier.

Moore, D. W., Anderson, A., Treccase, F., Deppeler, J., Furlonger, B., \& Didden, R. (2013). A video-based package to teach a child with autism spectrum disorder to write her name. Journal of Developmental and Physical Disabilities, 25(5), 493-503.

Morrison, G. R., Ross, S. M., Kemp, J. E., \& Kalman, H. (2010). Designing effective instruction: John Wiley \& Sons.

Nielsen, J., \& Nielsen, B. (1995). Multimedia and hypertext: The Internet and beyond: Morgan Kaufmann.

Norman, D. A. (2014). Some observations on mental models Mental models (pp. 15-22): Psychology Press. 
Ozonoff, S., \& Miller, J. N. (1995). Teaching theory of mind: A new approach to social skills training for individuals with autism. Journal of autism and developmental disorders, 25(4), 415-433.

Patel, V. L., Yoskowitz, N. A., Arocha, J. F., \& Shortliffe, E. H. (2009). Cognitive and learning sciences in biomedical and health instructional design: A review with lessons for biomedical informatics education. Journal of biomedical informatics, 42(1), 176-197.

Preece, J., \& Rombach, H. D. (1994). A taxonomy for combining software engineering and humancomputer interaction measurement approaches: towards a common framework. International Journal of Human-Computer Studies, 41(4), 553-583.

Putnam, C., \& Chong, L. (2008). Software and technologies designed for people with autism: what do users want? Paper presented at the Proceedings of the 10th international ACM SIGACCESS conference on Computers and accessibility.

Quill, K. A. (1997). Instructional considerations for young children with autism: The rationale for visually cued instruction. Journal of autism and developmental disorders, 27(6), 697-714.

Rada, R., \& Diaper, D. (1991). Converting text to hypertext and vice versa. Hypermedia/Hypertext and object-oriented databases, 167-200.

Robson, C., Blampied, N., \& Walker, L. (2015). Effects of feedforward video self-modelling on reading fluency and comprehension. Behaviour Change, 32(1), 46-58.

Sigafoos, J., O’Reilly, M., Cannella, H., Edrisinha, C., de la Cruz, B., Upadhyaya, M., .. . Garver, C. (2007). Evaluation of a video prompting and fading procedure for teaching dish washing skills to adults with developmental disabilities. Journal of Behavioral Education, 16(2), 93-109.

Speaks, A. (2011). What is autism. Retrieved on November, 17, 2011.

Tetreault, A. S., \& Lerman, D. C. (2010). Teaching social skills to children with autism using point-of-view video modeling. Education and treatment of children, 33(3), 395-419.

Umbach, P. D. (2004). Web surveys: Best practices. New directions for institutional research, 2004(121), 23-38.

Van Rijn, H., \& Stappers, P. J. (2008). The puzzling life of autistic toddlers: design guidelines from the LINKX project. Advances in Human-Computer Interaction, 2008.

Vora, P. R., \& Helander, M. G. (1997). Hypertext and its implications for the Internet Handbook of Human-Computer Interaction (Second Edition) (pp. 877-914): Elsevier. 
Whitlow, C. K., \& Buggey, T. (2003). Video self-modelling: an effective intervention for a preschooler with language delays. Journal of Research in Special Educational Needs, 3(1), no-no.

Wilson, A., \& Laskey, N. (2003). Internet based marketing research: a serious alternative to traditional research methods? Marketing Intelligence \& Planning, 21(2), 79-84.

Wise, R. (2014). 15 Behavior Strategies to Help Children with Autism. 